\title{
Intracytoplasmic sperm injection: promises and challenges
}

Tntracytoplasmic sperm injec1 tion (ICSI) was first developed in 1993 as a treatment for male factor infertility - specifically, to overcome problems with penetration of an oocyte by a sperm cell. It is estimated that 150000 couples sought medical help for infertility in 2002 in Canada; among them, 6400 underwent in vitro fertilization (IVF), in which ICSI was used in about one third of the IVF cycles. The live birth success rates for ICSI are similar to standard IVF $(24.1 \%$ and $23.7 \%) .{ }^{1}$ In Canada, the costs of standard IVF are about $\$ 6000$, and the additional costs for ICSI are approximately $\$ 1000$. Indications for ICSI are listed in Box 1.

In spite of the empiric success with ICSI, the long-term safety of this technique has not been conclusively established. Specifically, it is unclear whether ICSI is associated with a greater subsequent risk of birth defects.

Natural fertilization and conventional in vitro fertilization requires the successful completion of a number of steps. A fertilizing spermatozoon must pass layers of cells and then make contact with the zona pellucida, the acellular glycoproteinic envelope that surrounds and protects the oocyte. The interaction with the zona pellucida requires the recognition of specific receptors on both the sperm plasma membrane and the zona. To penetrate the zona, a spermatozoon must go through a process called the acrosome reaction, resulting in the release of enzymes that enable the digestion of a path through the zona. Once through the outer layers, the spermatozoon must then fuse with the plasma membrane of the oocyte and be incorporated into the oocyte. Fusion of the gametes triggers a cascade of events leading to the completion of the oocyte's meiosis and the formation of the male and female pronuclei.

Because ICSI involves the injection of a single spermatozoon directly into the oocyte (Fig. 1), this procedure bypasses all the preliminary steps of fertilization. Matured eggs are obtained from the woman by hormonal ovarian hyperstimulation. The "best" sperm are selected on the basis of morphology and mobility.

\section{Safety of ICSI}

It is easy to appreciate that ICSI is invasive to both the oocyte and the sperm; little imagination is required to conjecture that problems may arise from the mechanical disruption of the oocyte itself, resulting in potential birth defects not seen in natural fertilization or conventional IVF. For example, the final portion of oocyte maturation occurs after egg activation, and perturbations to the egg could alter maturation and activation and produce long-term consequences. De novo chromosomal anomalies (abnormal fetal karyotypes that are not inherited from either parent) are possible consequences of ICSI. In particular, errors that inap-

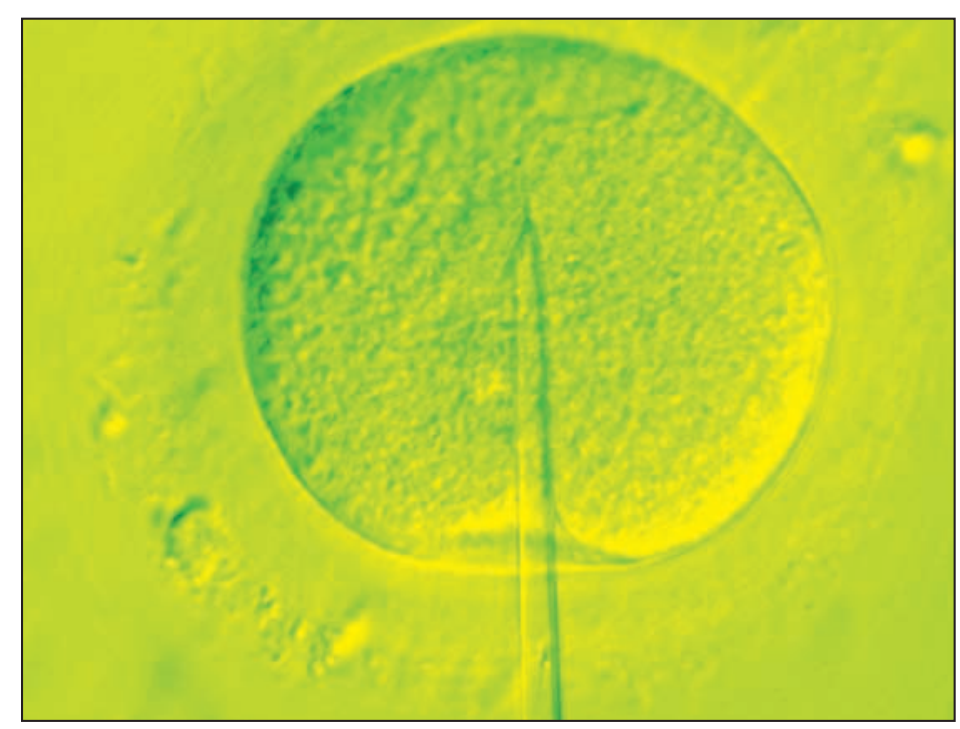

Giving sperm a helping hand.

\author{
Box 1: Indications for ICSI \\ Established indications \\ Severe deficit in semen quality \\ Obstructive azoospermia (failure \\ to ejaculate any sperm in the \\ presence of a mechanical \\ obstruction in the ejaculatory \\ system) \\ Nonobstructive azoospermia \\ (failure to ejaculate any sperm in \\ the absence of any mechanical \\ or physiological barrier) \\ Failed IVF or very poor \\ fertilization \\ Possible indications ${ }^{3}$ \\ Sperm count $<1$ million \\ Sperm morphology $<4 \%$ normal \\ forms
}

propriately activate a parental allele have been reported. ${ }^{4}$

The available epidemiologic evidence on the safety of ICSI for the fetus is inconsistent. A study in West Australia found that both ICSI and IVF were associated with increased major birth defects at 1 year compared with natural conception (odds ratio [OR] 2.0, 95\% conOR 2.0 , 95\% CI 1.5 to 2.9 respectively]. ${ }^{5}$ In contrast, a fidence interval [CI] 1.3 to 3.2 ; 
large-scale study in Sweden found that the incidence of malformations in children born after ICSI was not significantly higher than in normal controls. ${ }^{6}$ Although the OR for malformation was 1.75 (95\% CI 1.19-2.58) for ICSI after adjustment for delivery hospital, year of birth and maternal age, it was reduced to 1.19 (95\% CI 0.79-1.81) after adjustment for multiple births (a factor known to increase the risk of birth defects). In a casecontrolled study in the UK, Sutcliffe et al found no difference in mean neurodevelopmental scores or in any subscales on the Griffiths' scales of mental development ${ }^{3}$ between 208 singleton children conceived by ICSI and the 211 naturally conceived control group matched for social class, maternal educational attainment, region, sex, and race; perinatal outcomes were also similar between the 2 study groups. Bonduelle et al compared the outcomes of ICSI (2840 infants) and IVF (2955 infants) and found that the rate of prematurity was slightly higher in the ICSI $(31.8 \%)$ group than in the IVF $(29.3 \%)$ $(p<0.05)$ group, but the rate of very low birth weight was higher with IVF $(5.7 \%)$ than with ICSI $(4.4 \%)(p<0.05)$. The rates of major malformations at birth were similar between the 2 groups. ${ }^{7}$

The difficulty in using evidence from observational studies to determine the contribution of ICSI to the risk of birth defects lies in the difficulty in disentangling the contribution of the underlying infertility itself: birth defects are more common among offspring of couples who had difficulty in conceiving but were not treated for infertility. The nature of the link between infertility and increased risk of birth defects is unclear. It may be related to a sharing of common pathophysiologic factors underlying both infertility and adverse pregnancy outcomes, or to a sharing of risk factors that causes both infertility and adverse pregnancy outcomes. However, because of ethical concerns and practical difficulties, it is impossible to conduct randomized controlled trials to assess the long-term safety of ICSI.

Given that the evidence on the safety of ICSI is conflicting, opting for this treatment can present a difficult choice for patients. Physicians should counsel patients based on the best possible evidence available and then allow the couple to make an informed choice. The options of donor sperm insemination, adoption and remaining childless should always be discussed. Couples should feel free to make the choice that is acceptable to them given the information that is available. No one should feel pressured to pursue an infertility treatment.

In the future, large scale cohort studies that examine demographic, clinical and laboratory data may shed more light on ICSI as a risk factor for birth defects. Only by examining adverse fetal and infant outcomes simultaneously, and determining the association between ICSI and rare clinical conditions while controlling for confounding factors, can we provide the evidence badly needed for infertile couples to make an informed choice.
Shi Wu Wen

Mark C. Walker

Marie-Claude Léveillé

Art Leader

From the Division of Maternal-Fetal Medicine, Department of Obstetrics and Gynecology, University of Ottawa, and the Clinical Epidemiology Program, Ottawa Health Research Institute (Wen, Walker), the Division of Reproductive Medicine, Department of Obstetrics and Gynecology, University of Ottawa (Leader, Léveillé) and the Hormones, Growth and Development Program, Ottawa Health Research Institute (Leader, Léveillé), Ottawa, Ont.

This work was supported in part by a grant from the Program on Oocyte Health (www.ohri.ca/oocyte) funded under the CIHR's Healthy Gametes and Great Embryos Strategic Initiative, Child and Youth Health grant number HGG62293. Dr. Wen is a CIHR New Investigator, and Dr. Walker is a Career Scientist with the Ontario Ministry of Health and Long-Term Care.

\section{References}

1. Provisional National Data Statistics IVF from April 1999 to March 2000: Human Embryology and Fertility Authority. London(UK): 2002.

2. Ombelet W, Deblaere K, Bosmans E, Cox AM, Jacobs P, Janssen M, Nijs $M$. Semen quality and intrauterine insemination. Reprod BioMed. [online]. 2003;7(4):485-92.

3. Sutcliffe AG, Taylor B, Saunders K, Thornton S, Lieberman BA, Grudzinskas JG. Outcome in the second year of life after in-vitro fertilisation by intracytoplasmic sperm injection: a UK casecontrol study. Lancet 2001;357:2080-4.

4. Cox GF, Burger J, Lip V, Mau UA, Sperling K, Wu BL, et al. Intracytoplasmic sperm injection may increase the risk of imprinting defects. Am 7 Hum Genet 2002;71:162-4.

5. Hansen M, Kurinczuk JJ, Bower C, Webb S. The risk of major birth defects after intracytoplasmic sperm injection and in vitro fertilization. $N$ Engl 7 Med 2002;346:725-30.

6. Wennerholm UB, Bergh C, Hamberger L, Lundin K, Nilsson L, Wikland $\mathrm{M}$, et al. Incidence of congenital malformations in children born after ICSI. Hum Reprod 2000;15:944-8.

7. Bonduelle M, Liebaers I, Deketelaere V, Derde MP, Camus M, Devroey P et. Neonatal data on a cohort of 2889 infants born after ICSI (1991-1999) and of 2995 infants born after IVF (19831999). Hum Reprod 2002;17:671-94. 